\title{
Postoperative spinal subdural hygroma without incidental durotomy: illustrative cases
}

\author{
Garrett Q. Barr, BS, and Peter L. Mayer, MD \\ Florida State University College of Medicine, Department of Clinical Sciences, Sarasota, Florida
}

BACKGROUND Spinal subdural hygroma (SSH) is a rare pathological entity occurring as a complication of spinal surgery. It is different from spinal subdural hematoma due to blunt trauma, anticoagulation therapy, spinal puncture, and rupture of vascular malformations.

OBSERVATIONS The authors presented five patients with SSH who received decompression for lumbar stenosis. None had incidental durotomy. All presented postoperatively with unexpectedly severe symptoms, including back and leg pain and weakness. Postoperative magnetic resonance imaging (MRI) revealed SSH with a characteristic imaging finding termed the "flying bat" sign. Four patients underwent evacuation of SSH, with immediate and complete resolution of symptoms in three patients and improvement in one patient. One patient improved without additional surgery. At surgery, subdural collections were found to be xanthochromic fluid in three patients and plain cerebrospinal fluid (CSF) in one patient.

LESSONS Unexpectedly severe back and leg pain and weakness after lumbar or thoracic spine surgery should raise suspicion of SSH. MRI and/or computed tomography myelography shows the characteristic findings termed the flying bat sign. Surgical evacuation is successful although spontaneous resolution can also occur. The authors hypothesized that SSH is due to CSF entering the subdural space from the subarachnoid space via a one-way valve effect.

https://thejns.org/doi/abs/10.3171/CASE21291

KEYWORDS spinal subdural hygroma; spinal subdural effusion; spinal subdural hematoma

Spinal subdural hygroma (SSH) was first described by Schiller et al. 73 years ago. ${ }^{1}$ Various case reports and small series of spinal subdural hematomas associated with trauma, anticoagulation therapy, blood disorders, spinal puncture, and vascular malformation/neoplasm have been reported. ${ }^{2-7}$ In addition, SSH may develop as a complication of spinal surgery. ${ }^{8-15}$ In most postoperative cases reported previously, incidental durotomy was noted at the time of surgery. ${ }^{9-12}$ We present a series of five cases of acute/subacute SSH without incidental durotomy. To our knowledge, only one previous case report has been published in the literature describing this rare phenomenon. ${ }^{13}$

\section{Illustrative Cases}

All cases are summarized in Table 1.

\section{Case 1}

A 75-year-old man with several previous spine operations presented with recurrent lumbar stenosis and pseudarthrosis. He received L2-L5 removal of posterior instrumentation, L1-L2, L2-L3, L3-L4 lateral lumbar interbody fusion, minimally invasive L4-L5 and L5-S1 transverse lumbar interbody fusion, and T10-S1 posterolateral fusion and instrumentation.

Immediately postoperatively, the patient had intact bilateral lower extremity neurological function. However, on postoperative day 3 , bilateral leg weakness developed. Examination showed 1/5 weakness of quadriceps and 3-4/5 weakness of dorsiflexion and plantarflexion. Sensation was intact to light touch throughout. By postoperative day 4 , examination showed $2 / 5$ weakness of quadriceps, $2 / 5$ dorsiflexion, and $3 / 5$ plantarflexion. Light touch and proprioception were intact, but pinprick sensation was absent below T8.

A computed tomography (CT) scan showed no problematic postoperative findings. Thoracic and lumbar spine magnetic resonance imaging (MRI) showed spinal cord signal abnormality from T8 to T10, suggestive of infarction. Neurology and neurosurgery were consulted at this time.

Additional review of the lumbar MRI indicated a flying bat sign (Fig. 1A-D) throughout the lumbar spine, and SSH was suspected. Because of the unusual nature of the findings, a myelogram was

ABBREVIATIONS CSF = cerebrospinal fluid; $\mathrm{CT}=$ computed tomography; MRI = magnetic resonance imaging; $\mathrm{SSH}=$ spinal subdural hygroma . INCLUDE WHEN CITING Published December 6, 2021; DOI: 10.3171/CASE21291.

SUBMITTED May 9, 2021. ACCEPTED July 29, 2021.

(c) 2021 The authors, CC BY-NC-ND 4.0 (http://creativecommons.org/licenses/by-nc-nd/4.0/). 
TABLE 1. Patient summary

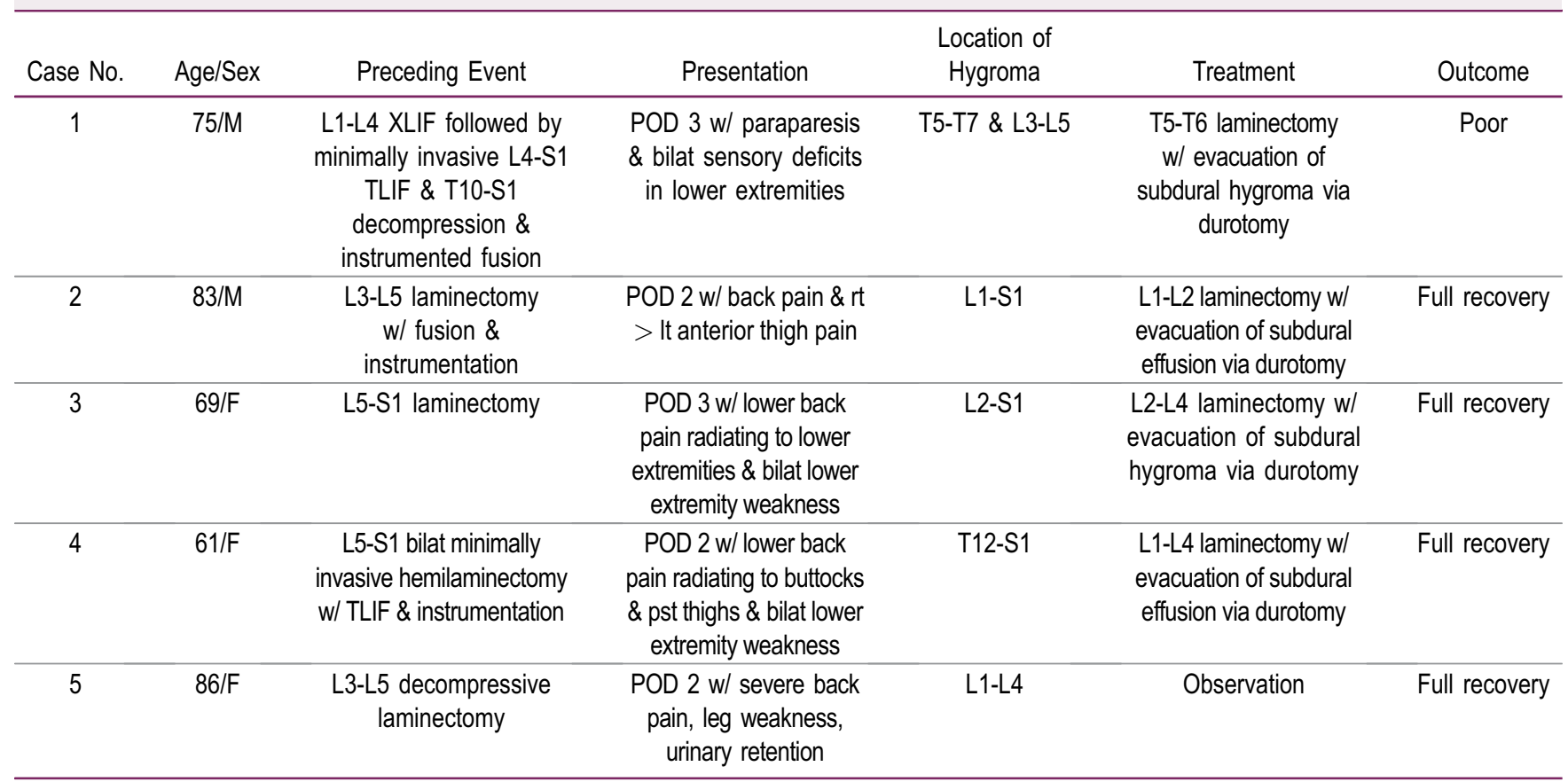

$\mathrm{POD}=$ postoperative day; pst = posterior; TLIF = transverse lumbar interbody fusion; XLIF = lateral lumbar interbody fusion.

also obtained. It revealed a different picture from MRI. A large thoracic $\mathrm{SSH}$ tracked throughout the thoracic and lumbar spine, with mass effect mainly at T5-T7 (Fig. 1E and F).

The patient received a T5-T6 laminectomy with drainage of $\mathrm{SSH}$. At the time of surgery, the dura was tense, with a mottled, bluish discoloration. Under the operating microscope, a small midline durotomy was performed and immediately, a gush of thin xanthochromic fluid came forth under pressure. Various instruments were used to push the arachnoid down, and Valsalva maneuvers were performed, allowing further egress of xanthochromic subdural fluid. At the end of the subdural decompression, the arachnoid was opened to reveal crystal-clear cerebrospinal fluid (CSF), confirming that the two spaces were, in fact, separate. Postoperative MRI confirmed decompression of the spinal cord and normalization of the subdural space (Fig. $1 \mathrm{G}$ and $\mathrm{H}$ ). Postoperatively, the patient experienced immediate partial improvement of neurological function.

\section{Case 2}

An 83-year-old man presented with severe lumbar stenosis and neurogenic claudication. He underwent L3-L5 laminectomy, posterolateral fusion, and instrumentation. Postoperatively, the patient felt well for 1 day. On postoperative day 2, he reported new anterior right thigh pain. Over the next several weeks, he experienced progressively severe back and thigh pain and recurrence of neurogenic claudication.

Three weeks postoperatively, a repeat lumbar spine MRI showed $\mathrm{SSH}$ from L1 to S1 (Fig. 2A-F) with clumping of the nerve roots of the cauda equina. The axial cross-section of clumped nerve roots (Fig. 2B and I) we refer to as the flying bat sign. Notably, in this case, the largest SSH collection was at L1-L2, which was superior to the levels of the original decompression. Also, because this maximal area of subdural fluid collection was at the level of the conus medullaris rather than the cauda equina, axial MRI showed compression of the conus without a true flying bat sign (Fig. $2 \mathrm{C}$ and $\mathrm{D}$ ).

The patient received L1-L2 laminectomy with drainage of SSH. At surgery, when the dura was opened, a gush of crystal-clear fluid (i.e., loculated CSF) drained out under significant pressure. The dura was opened farther, and the intact normal arachnoid layer bulged out. The arachnoid was then fenestrated to allow full communication between the subdural and subarachnoid spaces, and the dura was closed again. Postoperatively, the back and leg symptoms resolved immediately. An MRI scan obtained after the second surgery showed normalization of the thecal sac and nerve roots (Fig. $2 \mathrm{G}$ and $\mathrm{H}$ ).

\section{Case 3}

A 69-year-old woman presented with severe L5-S1 stenosis with radicular pain and neurogenic claudication. She received L5-S1 laminectomy. She did well at first and was discharged home on postoperative day 1 . On postoperative day 2, the patient developed recurrent back and leg pain. On postoperative day 3 , she presented to the emergency department by ambulance for severe back and leg pain and inability to stand or walk. Examination showed 3/5 weakness of the bilateral lower extremities with diminished light tough and pinprick sensations and loss of reflexes. Lumbar spine MRI revealed SSH from L1 to $L 5$ with severe compression of the nerve roots of the cauda equina (i.e., flying bat sign).

An L2-L5 laminectomy was performed with evacuation of SSH. At the time of surgery, the dura was full, with a mottled, slightly blue discoloration. The dura was opened, and thin xanthochromic fluid came forth under pressure. Postoperatively, the patient's symptoms resolved immediately. 


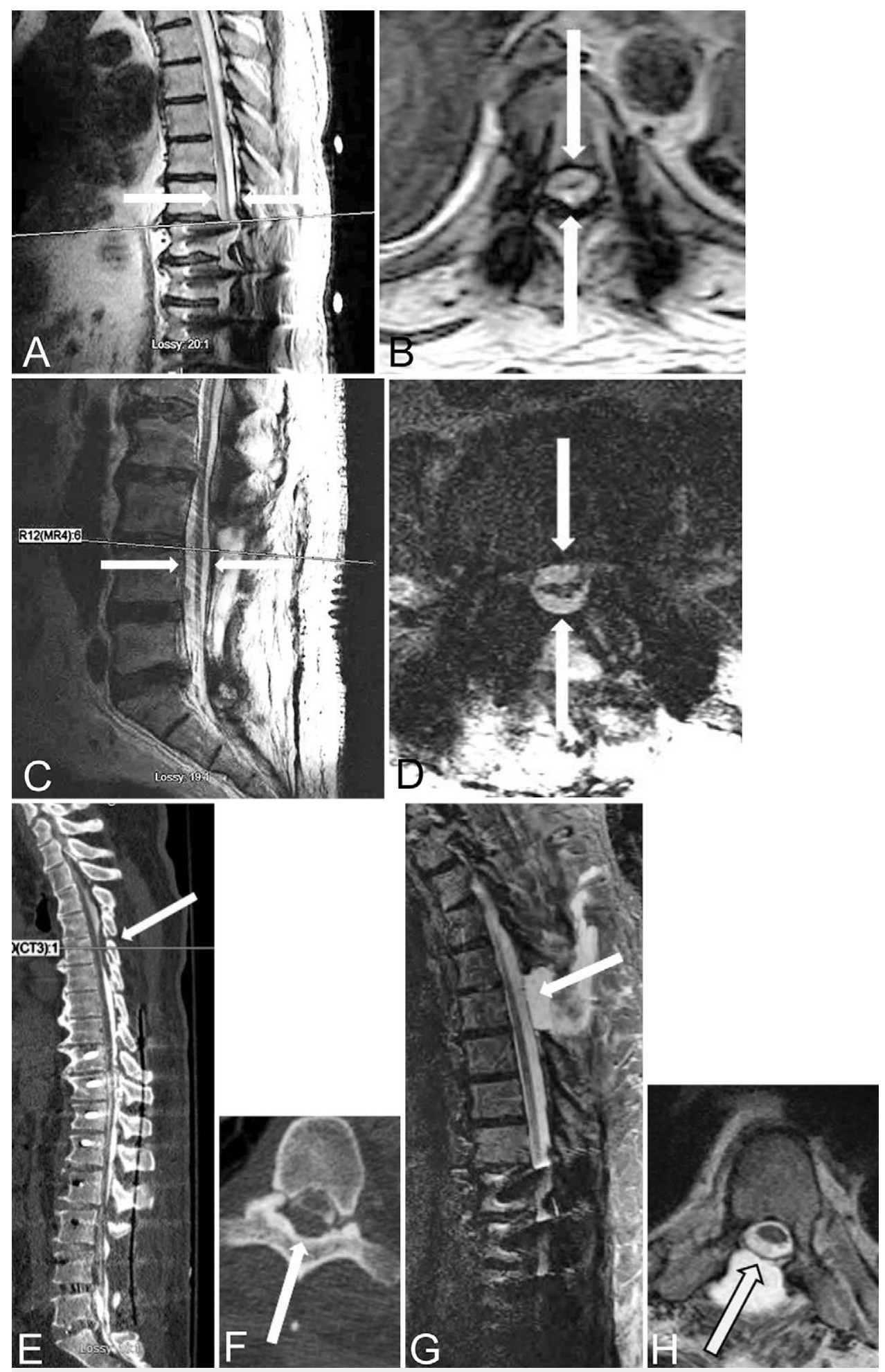

FIG. 1. Case 1. Sagittal (A and C) and axial (B and D) MRI after first operation showing SSH (white arrows). Axial images resemble a stylized drawing of a flying bat (D, arrows). Sagittal $(\mathbf{E})$ and axial $(\mathbf{F})$ CT myelogram after first operation showing SSH (white arrow). Sagittal (G) and axial (H) MRI showing subsequent resolution of SSH at the same levels (white arrow). 


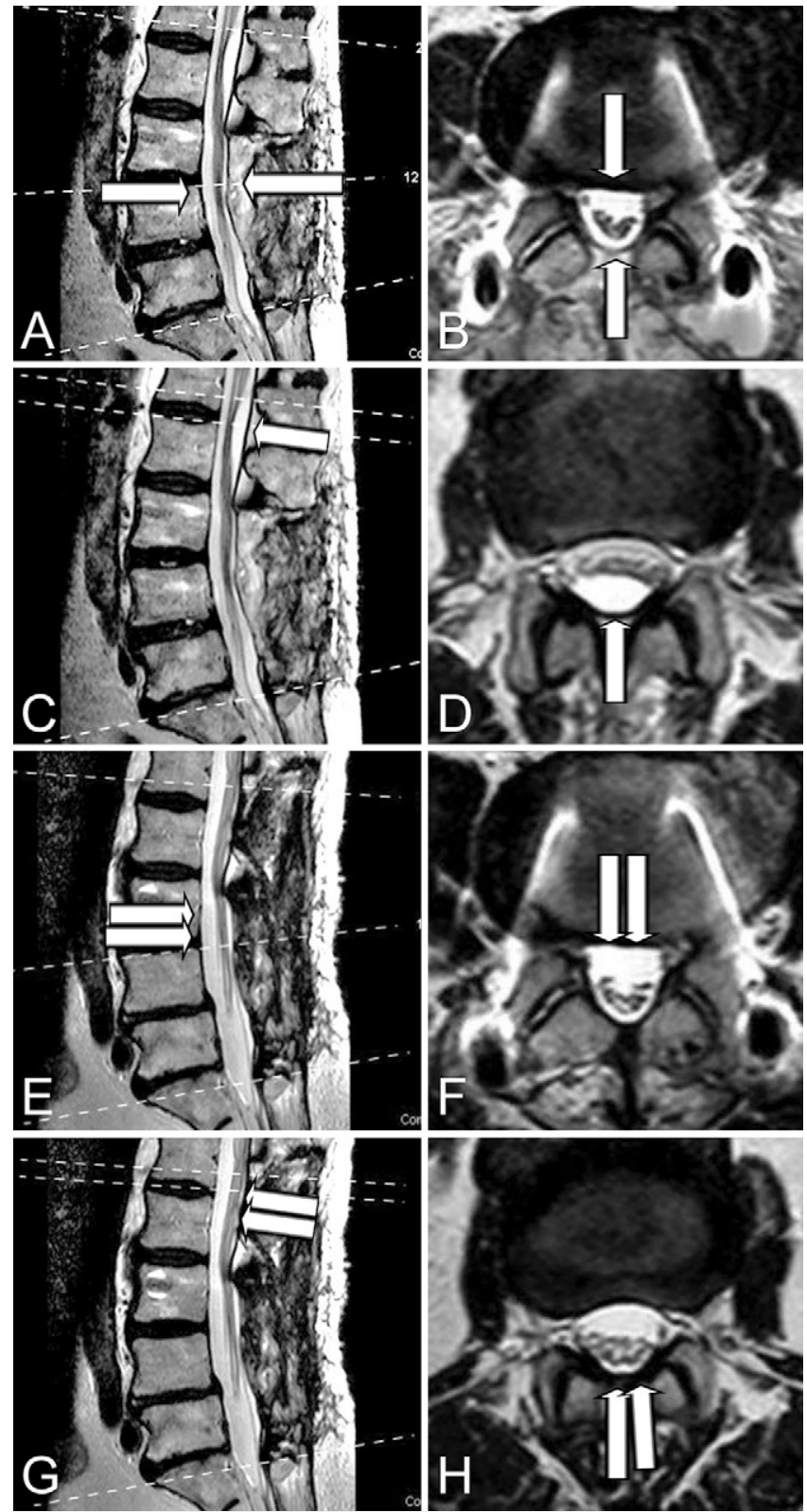

FIG. 2. Case 2. Sagittal (A, C, E, F) and axial (B, D, F, H) MRI after first $(\mathbf{A}-\mathbf{D})$ and second $(\mathbf{E}-\mathbf{H})$ operations showing SSH (A-D, white single arrows) and subsequent resolution of SSH at the same levels $(\mathrm{E}-\mathrm{H}$, white double arrows). Axial image of SSH in B resembles a stylized image of a flying bat.

\section{Case 4}

A 61-year-old woman who had received right L5-S1 laminotomy and microdiscectomy 3 years prior to admission presented with worsening symptoms secondary to spinal stenosis and degenerative disc disease. The patient received surgery for bilateral, minimally invasive L4-L5 and L5-S1 decompression, with L5-S1 transverse lumbar interbody fusion. Postoperatively, she developed unexpectedly severe low back and posterior leg pain. Neurological examination showed moderate bilateral lower extremity weakness with difficulty standing or walking.
Lumbar MRI showed an SSH from T12 to S1 with severe mass effect on the nerve roots of the cauda equina and a flying bat sign. The patient received repeat surgery with L1-L4 laminectomy, durotomy, and evacuation of the SSH. At the time of surgery, the dura was noted to be full and slightly discolored. The dura was opened minimally, and thin xanthochromic fluid came forth under pressure. The patient's symptoms resolved immediately postoperatively.

\section{Case 5}

An 86-year-old right-handed woman with mild dementia presented with severe lumbar stenosis with neurogenic claudication. She received L3 through L5 decompressive laminectomy. On postoperative day 1 , the patient noted minimal pain and marked improvement of neurogenic claudication. She was able to stand and walk with minimal assistance and was discharged home. However, the following day, the patient developed increased pain and leg weakness such that she fell in her bathroom. She was taken to the emergency department by her family and was admitted for further evaluation. On examination there was mild dementia, with mild bilateral $4 / 5$ weakness in the lower extremities. Sensory examination was intact to light touch and pinprick sensation throughout, including the perineum. There was mild urinary retention of $650 \mathrm{~mL}$.

Lumbar spine MRI revealed a small epidural hematoma at the site of surgery and a moderate SSH that tracked superiorly two additional levels. The two fluid collections could be clearly differentiated by virtue of their different anatomical compartments and MRI signals.

Based on the presentation and imaging, we determined that emergency surgical exploration was not needed, and the patient was scheduled for surgical evacuation of SSH the next day. However, the patient's symptoms resolved spontaneously prior to surgery and returned to her previous postoperative baseline. She was discharged home again and remained neurologically intact thereafter.

\section{Discussion}

\section{Observations}

Spinal subdural hygroma is a rare complication of decompressive surgery for spinal stenosis. It presents as unexpectedly severe back and leg pain and neurological deficits. Imaging, including MRI and myelography, reveals characteristic findings. In the lumbar spine there is clumping of the nerve roots of the cauda equina and mass effect on the conus medullaris. Patients present with cauda equina or conus medullaris syndrome. In the thoracic spine, imaging reveals spinal cord compression, and patients present with myelopathy. In one of our cases, spinal cord infarction was present. The cause of neurological embarrassment is the high-pressure accumulation of fluid in the confined spinal subdural space, resulting in severe compression of neural and vascular elements.

\section{Pathophysiology}

Various hypotheses have been suggested to explain the pathophysiology of SSH. One group has proposed that SSH originates secondary to a rapid increase in intrathoracic and/or intraabdominal pressure. $^{16}$ It increases the intravascular pressure within the spinal subdural and subarachnoid spaces and may lead to the rupture of veins lining the inner surface of the dura or the larger vessels in the subarachnoid space. ${ }^{7,15,16}$ This hypothesis may be supported by reports of SSH arising as a complication of vertebroplasty, possibly secondary to venous congestion. ${ }^{16}$ These authors propose that if bleeding occurs within the subarachnoid space and becomes 
sufficiently profuse, it may rupture through the arachnoid and into the subdural space. ${ }^{7,17,18}$ Cases of simultaneous spinal subarachnoid and subdural hemorrhage have also been reported. ${ }^{18-20}$ These authors theorize that given the diluting and redistributing effect of CSF, the hemorrhage within the subarachnoid space may be washed away, leaving only the $\mathrm{SSH}^{21}$

SSH has also been shown to be a result of traumatic introduction of blood into the subdural space by a traumatic lumbar puncture. $^{22,23}$ In these cases, postmortem examination found laceration of radicular vessels to be the source of the bleeding, which was traumatically introduced into the subdural space. However, in these cases there is true hematoma, not hygroma. Another hypothesis contends that SSH may be the result of the downward displacement of an intracranial subdural hemorrhage. Electron microscopy has demonstrated that the intracranial and spinal subdural space is continuous, and cases have been reported of coexisting spinal and intracranial subdural hematomas. ${ }^{24-26}$ Like ours, however, most cases do not suggest cranial subdural hematoma.

Previous authors have suggested that SSH is likely a result of incidental durotomy ${ }^{9-12}$ although cases also have been reported of postoperative SSH without incidental durotomy., ${ }^{8,13}$ In cases in which an incidental durotomy occurred, a small tear in the arachnoid has been described with the presumed formation of a one-way valve mechanism that allows CSF to enter the subdural space. ${ }^{12}$

In our opinion, it is most likely that the subdural fluid in $\mathrm{SSH}$ is CSF that may or may not contain a small amount of blood. We propose that the act of decompressing the thecal sac results in arachnoidal trauma, leading to a one-way valve phenomenon whereby CSF enters the subdural space. With Valsalva maneuver and normal motion of the spine, CSF enters the subdural space, which progressively enlarges while the subarachnoid space diminishes until the subdural space cannot further accommodate increases in fluid. Increasing fluid in a loculated space causes pressure increase that is transmitted to the neural and vascular elements, which results in neurological compromise and ischemia. As the SSH collects, subdural fluid can distribute posterior or anterior to the neural elements or both. Because the arachnoid is anchored laterally by the denticulate ligaments, ${ }^{14}$ the compartmentalization of $\mathrm{SSH}$ results in the characteristic imaging findings on axial MRI or axial CT myelography (i.e., the nerve roots of the cauda equina are seen clumped and compressed with pointed irregularities). We refer to this finding as the "flying bat sign" because of its similarity to the silhouetted shape of a flying bat. Others have described the finding as similar to an inverted or partially inverted Mercedes-Benz logo. ${ }^{14}$

\section{Terminology}

Several authors have reported cases similar to ours, and various terminology has been used to describe the findings of SSH. The terms spinal subdural hematoma, effusion, and hygroma have all

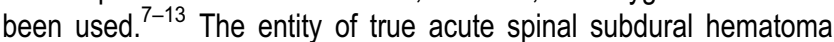
has been reported, ${ }^{1,3,4,8,15,19}$ most commonly in trauma, in association with anticoagulation, ${ }^{3,27}$ or as a result of hemorrhage of vascular malformation. ${ }^{28}$ True acute spinal subdural hematoma after spinal surgery has also been reported and is occasionally labeled as $\mathrm{SSH}^{29}$ However, these cases are different from what we are reporting here because they represent frank hemorrhage and clotted blood seen on MRI or at the time of surgery, whereas our cases do not. It is also not likely that the SSH fluid is an effusion (i.e., an exudate or secretion) because no physiological process can be invoked to explain these possibilities. Because the fluid in $\mathrm{SSH}$ is CSF, sometimes blood-tinged, we recommend the term spinal subdural hygroma to describe this phenomenon.

\section{Lessons}

Spinal subdural hygroma is a rare complication of decompression for spinal stenosis. Clinically, it is characterized by unexpectedly severe pain and neurological deficits, which can include radiculopathy, neurogenic claudication, cauda equina syndrome, and myelopathy. Imaging with either MRI or CT myelography reveals characteristic findings, including the flying bat sign on axial views. Subdural fluid may track up or down the subdural space and may even be found to be most problematic at sites distant from the initial surgery. Spontaneous resolution is possible, but surgical treatment is indicated to avoid permanent neurological deficits. ${ }^{25,27}$ Laminectomy with durotomy and evacuation of the effusion is the procedure of choice. Prognosis is good as long as the fluid is evacuated before severe compression causes permanent neurological damage or infarction.

\section{References}

1. Schiller F, Neligan G, Budtz-Olsen O. Surgery in haemophilia: a case of spinal subdural haematoma producing paraplegia. Lancet. 1948;2(6535):842-845.

2. Abla AA, Oh MY. Spinal chronic subdural hematoma. Neurosurg Clin N Am. 2000;11(3):465-471.

3. Bernsen RA, Hoogenraad TU. A spinal haematoma occurring in the subarachnoid as well as in the subdural space in a patient treated with anticoagulants. Clin Neurol Neurosurg. 1992;94(1):35-37.

4. Chen HJ, Liang CL, Lu K, Liliang PC, Tsai YD. Cauda equina syndrome caused by delayed traumatic spinal subdural haematoma. Injury. 2001;32(6):505-507.

5. Gambacorta D, Reale F, De Falco D. Spontaneous chronic spinal subarachnoid hematoma. Report of a case and review of the literature. Spine (Phila Pa 1976). 1987;12(7):716-718.

6. Kotwica Z, Stawowy A, Polis L. Spinal chronic subdural haematoma in a 7-year-old girl. Eur J Pediatr. 1989;148(8):779-780.

7. Russell NA, Benoit BG. Spinal subdural hematoma. A review. Surg Neurol. 1983;20(2):133-137.

8. Boe CC, Freedman BA, Kumar R, Lee K, McDonald R, Port J. Spinal subdural hematoma: a rare case of spinal subdural hematoma complicating routine, minimally invasive lumbar discectomy and decompression and relevant literature review. J Spine Surg. 2017;3(1):112-118.

9. Chang KC, Samartzis D, Luk KD, Cheung KM, Wong YW. Acute spinal subdural hematoma complicating lumbar decompressive surgery. Evid Based Spine Care J. 2012;3(1):57-62.

10. Gehri R, Zanetti M, Boos N. Subacute subdural haematoma complicating lumbar microdiscectomy. J Bone Joint Surg Br. 2000;82(7):1042-1045.

11. Reinsel TE, Goldberg E, Granato DB, Wilkinson S, Penn R. Spinal subdural hematoma: a rare cause of recurrent postoperative radiculopathy. J Spinal Disord. 1993;6(1):62-67.

12. Singleton WG, Ramnarine D, Patel N, Wigfield C. Post-operative spinal subdural extra-arachnoid hygroma causing cauda equina compression: a report of two cases. Br J Neurosurg. 2012;26(3): 429-431.

13. Elder BD, Ishida W, Goodwin RC, Bydon A. latrogenic spinal subdural extra-arachnoid hygroma following uncomplicated lumbar decompression. Cureus. 2017;9(4):e1171.

14. Pierce JL, Donahue JH, Nacey NC, et al. Spinal hematomas: what a radiologist needs to know. Radiographics. 2018;38(5):1516-1535.

15. Edelson RN, Chernik NL, Posner JB. Spinal subdural hematomas complicating lumbar puncture. Arch Neurol. 1974;31(2):134-137. 
16. Mattei TA, Rehman AA, Dinh DH. Acute spinal subdural hematoma after vertebroplasty: a case report emphasizing the possible etiologic role of venous congestion. Global Spine J. 2015;5(5): e52-e58.

17. Rader JP. Chronic subdural hematoma of the spinal cord: report of a case. N Engl J Med. 1955;253(9):374-376.

18. Kakitsubata Y, Theodorou SJ, Theodorou DJ, et al. Spontaneous spinal subarachnoid hemorrhage associated with subdural hematoma at different spinal levels. Emerg Radiol. 2010;17(1):69-72.

19. Kyriakides AE, Lalam RK, El Masry WS. Acute spontaneous spinal subdural hematoma presenting as paraplegia: a rare case. Spine (Phila Pa 1976). 2007;32(21):E619-E622.

20. Vinters HV, Barnett HJ, Kaufmann JC. Subdural hematoma of the spinal cord and widespread subarachnoid hemorrhage complicating anticoagulant therapy. Stroke. 1980;11(5):459-464.

21. Sneyers B, Ramboer K. Spinal subdural hygroma. Acta Neurol Belg. 2021;121(2):311-319.

22. Breuer AC, Tyler HR, Marzewski DJ, Rosenthal DS. Radicular vessels are the most probable source of needle-induced blood in lumbar puncture: significance for the thrombocytopenic cancer patient. Cancer. 1982;49(10):2168-2172.

23. Masdeu JC, Breuer AC, Schoene WC. Spinal subarachnoid hematomas: clue to a source of bleeding in traumatic lumbar puncture. Neurology. 1979;29(6):872-876.

24. Nicholas DS, Weller RO. The fine anatomy of the human spinal meninges. A light and scanning electron microscopy study. J Neurosurg. 1988;69(2):276-282.

25. Hung KS, Lui CC, Wang CH, Wang CJ, Howng SL. Traumatic spinal subdural hematoma with spontaneous resolution. Spine (Phila Pa 1976). 2002;27(24):E534-E538.

26. Bortolotti C, Wang H, Fraser K, Lanzino G. Subacute spinal subdural hematoma after spontaneous resolution of cranial subdural hematoma: causal relationship or coincidence? Case report. J Neurosurg. 2004;100(4 Suppl Spine):372-374.

27. Jimbo H, Asamoto S, Mitsuyama T, Hatayama K, Iwasaki Y, Fukui Y. Spinal chronic subdural hematoma in association with anticoagulant therapy: a case report and literature review. Spine (Phila $\mathrm{Pa}$ 1976). 2006;31(6):E184-E187.

28. Caughey W, Manners S. Spinal subdural, extra-arachnoid hygroma following lumbar decompression: a rare case following cauda equina syndrome. ANZ J Surg. 2020;90(12):2567-2569.

29. Han PP, Theodore N, Porter RW, Detwiler PW, Lawton MT, Spetzler RF. Subdural hematoma from a Type I spinal arteriovenous malformation. Case report. J Neurosurg. 1999;90(2 suppl):255-257.

\section{Disclosures}

The authors report no conflict of interest concerning the materials or methods used in this study or the findings specified in this paper.

\section{Author Contributions}

Conception and design: Mayer. Acquisition of data: Mayer. Analysis and interpretation of data: both authors. Drafting the article: both authors. Critically revising the article: both authors. Reviewed submitted version of manuscript: both authors. Approved the final version of the manuscript on behalf of both authors: Barr. Study supervision: Mayer.

\section{Correspondence}

Garrett Q. Barr: Florida State University College of Medicine, Sarasota, FL.gqb10@med.fsu.edu. 